\title{
Avaliação dos resultados neonatais e fatores associados em gestantes com pré-eclâmpsia grave: uma revisão sistemática
}

\author{
Evaluation of neonatal outcomes and associated factors in pregnant women with severe \\ preeclampsia: a systematic review
}
Evaluación de los resultados neonatales y factores asociados en pacientes con preeclampsia grave: una revisión sistemática

Wyrna Schwenck de Almeida ${ }^{1}$, Fernanda Garcia Marzagão ${ }^{1}$, Jaqueline Gabriele Silva ${ }^{1}$, Jussara Nylma Macedo de Assis ${ }^{1}$, Rachel Laguardia Rego ${ }^{1}$, Rodolfo Lucas Silva Mourato ${ }^{1}$, Lívia Carolina Andrade Figueiredo ${ }^{1}$, Saulo Nascimento de Melo ${ }^{1}$, Vinícius Silva Belo ${ }^{1}$, Leonardo Lopes Tonani ${ }^{1 *}$.

\section{RESUMO}

Objetivo: Analisar o impacto da pré-eclâmpsia (PE) grave nas mortalidades materna e neonatal e fatores associados em gestantes portadoras. Métodos: Revisão de literatura incluindo estudos publicados entre 2014 e 2019, realizada na base de dados Medical Literature Analysis and Retrieval System Online (MEDLINE) via Pubmed em outubro de 2019. Mediante os descritores mortalidade neonatal, pré-eclâmpsia e mortalidade materna, inicialmente, encontrou-se 1669 estudos. Desses, 16 analisaram gestantes de qualquer idade e etnia com PE grave e foram selecionados para a revisão. Resultados: As mortalidades neonatal e materna apresentaram medianas de proporções iguais a $9.32 \%$ e $1 \%$, respectivamente. Sobre os resultados neonatais: a mediana de recém-nascidos (RNs) prematuros encontrada foi de $33.9 \%$, a mediana de peso ao nascer de $2042.65 \mathrm{~g}$ (classificada como baixo peso) e a mediana da porcentagem dos RNs internados em UTI de $46.6 \%$. Observa-se que a mortalidade materna foi abordada em apenas 7 dos artigos analisados (43.75\% do total de artigos selecionados). Considerações finais: A presente revisão sistemática conclui que a PE grave apresenta impacto relevante na mortalidade neonatal e nos demais resultados neonatais avaliados. A respeito da mortalidade materna, há uma lacuna de produções científicas datadas no período analisado que busquem suas associações diretas com a PE grave.

Palavras-chave: Mortalidade neonatal, Pré-eclâmpsia, Mortalidade materna.

\begin{abstract}
Objective: To analyze the impact of severe pre-eclampsia (PE) on maternal and neonatal mortality and associated factors in pregnant women. Methods: Literature review with inclusion of studies published between 2014 and 2019, carried out in the Medical Literature Analysis and Retrieval System Online (MEDLINE) database via Pubmed in October 2019. Through the descriptors neonatal mortality, preeclampsia and maternal mortality, 1669 studies were found in the initial search. Of these, 16 analyzed pregnant women of any age and ethnicity with severe PE and were selected for review. Results: Neonatal and maternal mortality showed medians of proportions equal to $9.32 \%$ and $1 \%$, respectively. About neonatal results: the median of premature newborns (NBs) found was $33.9 \%$, the median birth weight was $2042.65 \mathrm{~g}$ (classified as low weight) and the median percentage of newborns admitted to the ICU was $46.6 \%$. It is observed that maternal mortality was addressed in only 7 of the analyzed articles $(43.75 \%$ of the total selected articles). Final considerations: This systematic review concludes that severe PE has a relevant impact on neonatal mortality and on the other assessed neonatal outcomes. Regarding maternal mortality, there is a gap in scientific productions in the analyzed period that seek their direct correlations with severe PE.
\end{abstract}

Keywords: Neonatal mortality, Preeclampsia, Maternal mortality.

${ }^{1}$ Universidade Federal de São João Del-Rei (USFJ), Divinópolis - MG. *E-mail: leotonani@hotmail.com 


\section{RESUMEN}

Objetivo: Analizar el impacto de la preeclampsia (PE) grave en la mortalidad materna y neonatal y los factores asociados en mujeres embarazadas. Métodos: Revisión de la literatura con inclusión de estudios publicados entre 2014 y 2019, realizada en la base de datos Medical Literature Analysis and Retrieval System Online (MEDLINE) através de Pubmed en octubre de 2019. A través de los descriptores mortalidad neonatal, preeclampsia y mortalidad materna, se encontraron 1669 estudios en la búsqueda inicial. De estos, 16 analizaron a mujeres embarazadas de cualquier edad y origen étnico con PE grave y fueron seleccionadas para su revisión. Resultados: La mortalidad neonatal y materna mostró medianas de proporciones iguales a $9.32 \%$ y $1 \%$, respectivamente. Sobre los resultados neonatales: la mediana de prematuros (RN) encontrados fue $33.9 \%$, la mediana del peso al nacer fue de $2042.65 \mathrm{~g}$ (clasificado como bajo peso) y la mediana del porcentaje de RN ingresados en $\mathrm{UCl}$ fue de $46.6 \%$. Se observa que la mortalidad materna fue tratada en solo 7 de los artículos analizados $(43.75 \%$ del total de artículos seleccionados). Consideraciones finales: Esta revisión sistemática concluye que la PE grave tiene un impacto relevante en la mortalidad neonatal y en los otros resultados neonatales evaluados. Con respecto a la mortalidad materna, existe una brecha en las producciones científicas fechadas en el período analizado que buscan sus correlaciones directas con la PE grave.

Palabras clave: Mortalidad neonatal, Preeclampsia, Mortalidad materna.

\section{INTRODUÇÃO}

A pré-eclâmpsia (PE) é uma síndrome hipertensiva da gestação, descrita como uma desordem decorrente da má perfusão placentária e da disfunção endotelial, que gera elevação dos níveis pressóricos ou proteinúria. Sua ocorrência se dá, geralmente, após a $20^{a}$ semana de gestação em gestantes previamente normotensas (DE OLIVEIRA ACM, et al., 2016). A frequência de PE mundial é de 2 a $8 \%$ (KAHHALE S, 2018). No Brasil, é a primeira causa de morte materna direta, sendo as maiores proporções identificadas nas regiões Norte e Nordeste, o que traduz a grande relevância epidemiológica da PE em Ginecologia e Obstetrícia no país (SOCIEDADE BRASILEIRA DE CARDIOLOGIA, 2009).

A PE pode ser classificada em formas leve e grave. A forma grave é diagnosticada pela elevação da pressão arterial sistólica a valores maiores ou iguais a $160 \mathrm{mmHg}$ e/ou diastólica a valores maiores ou iguais a $110 \mathrm{mmHg}$, associada ou não à proteinúria grave, acima de $2 \mathrm{~g}$ nas últimas 24 horas. Além disso, podem ocorrer sintomas visuais ou cerebrais persistentes, que impactam em uma maior morbi-mortalidade materno-fetal. Já a PE leve também está associada a um quadro de aumento pressórico e/ou proteinúria, mas não preenche os critérios para ser considerada grave (ROCELLA EJ, 2000).

De acordo com a Federação Internacional de Ginecologistas e Obstetras (FIGO), a PE constitui uma das principais causas de morbimortalidade materna e perinatal em todo o mundo (POON LC, et al., 2019). A insuficiência placentária e a antecipação do parto decorrentes dessa condição, muitas vezes, geram importantes repercussões ao feto e ao neonato, como elevadas taxas de mortalidade peri e neonatal (FEBRASGO, 2017). Avaliando-se as consequências da PE para o feto, tem-se a prematuridade do recémnascido (RN) como uma complicação frequente, seja por decorrência de trabalho de parto espontâneo ou por conduta obstétrica de interrupção da gravidez, em razão de comprometimento materno-fetal. A prematuridade aumenta a taxa de morbidade e mortalidade perinatais, com possibilidade de sequelas imediatas ou tardias (CHAIM SRP, 2008).

Outros resultados neonatais negativos se relacionam com a PE, como score de APGAR baixo. Esse score é considerado baixo caso seja menor do que 7, o que indica necessidade de atenção especial ao RN. A idade gestacional, malformações do RN e condições maternas, como uso de medicamentos, têm sido descritos como fatores que podem influenciar o valor obtido no score (DE OLIVEIRA TG, et al., 2012). Outro parâmetro relevante no que diz respeito à morbimortalidade neonatal é o baixo peso ao nascer (BPN). Juntamente com o escore de APGAR e a necessidade de internação em UTI, este é um parâmetro que mede o bem-estar e o sucesso das atenções pré, peri e neonatal (TOURINHO AB, 2013). 
Ante o exposto, considerando que a PE, principalmente em sua forma grave, constitui-se um fator associado a piores resultados neonatais e tendo em vista a escassez de revisões sistemáticas sobre esse tema, a presente revisão analisa as mortalidades neonatal e materna e descreve os possíveis resultados neonatais adversos em gestantes com PE grave.

\section{MÉTODOS}

Foi realizada, em outubro de 2019, uma revisão da literatura a respeito do impacto da PE grave na mortalidade materna e neonatal. Foram selecionadas publicações sobre o tema, por meio de uma busca na base de dados MEDLINE, via Pubmed. O período para inclusão de estudos foi definido entre os anos de 2014 e 2019, com o objetivo de abranger a literatura com critérios diagnósticos e protocolos de manejo de PE recentes, permitindo uma melhor padronização dos resultados. A descrição desta revisão baseou-se na diretriz Preferred Reporting Items for Systematic Reviews (PRISMA) (THE PRISMA GROUP, 2015).

Como critérios de inclusão, foram selecionados estudos primários, disponíveis em texto completo, de quaisquer países de origem, envolvendo gestantes de qualquer idade e etnia, com PE grave e seus resultados neonatais. Quanto ao desenho metodológico dos estudos, foram considerados os estudos transversais, de caso controle, ensaios clínicos, séries de casos e estudos de coorte. A PE grave foi definida de acordo com os critérios do American College of Obstetricians and Gynecologists (ACOG) (WISNER, 2019). Foram excluídos estudos que envolvessem mulheres com PE leve, com menos de 20 semanas de gestação ou quando não eram descritos os critérios diagnósticos de PE grave, além de revisões sistemáticas e relatos de caso.

Para as buscas, foram utilizados os seguintes descritores: "preeclampsia" AND "neonatal mortality" AND "maternal mortality". Não houve restrição de idioma. Primeiramente, foram selecionados pelo título os artigos considerados potencialmente relevantes e compatíveis com os objetivos da revisão. Em seguida, dois pesquisadores independentes elegeram os resumos dos artigos que estivessem de acordo com os critérios de inclusão e de exclusão e que teriam lido o texto completo. A leitura dos estudos na íntegra foi realizada para garantir que as publicações incluídas estivessem de acordo com o objetivo geral desta revisão e com os critérios adotados. Referências de revisões sistemáticas relevantes também foram utilizadas na busca.

Os dados coletados foram extraídos no programa Microsoft Excel 15.0 (Office 2013), com campos relativos a: título do estudo, ano de publicação, local (cidade/país), nome do primeiro autor, desenho do estudo, número total de gestantes, número de gestantes com PE grave, mortalidade materna, mortalidade neonatal e resultados neonatais. Haja vista a diversidade de populações estudadas e de critérios adotados, os resultados obtidos foram apresentados de modo teórico, sem a condução de meta-análises para combinação das proporções. Procedeu-se, então, uma análise descritiva da amostra de publicações, também realizada por meio do programa Microsoft Excel.

Foi realizada análise da qualidade dos artigos de acordo com os critérios das diretrizes Strengthening the Reporting of Observational Studies in Epidemiology (STROBE) 2007 (PACHECO RL, 2017). Na presente revisão, definiu-se que a pontuação total da ferramenta poderia variar de zero a 22, sendo um ponto atribuído para cada critério cumprido. Para classificação da qualidade dos estudos, foram utilizados os critérios de Mataratzis PSR, et al. (2010). Segundo os autores, por meio do STROBE é possível agrupar os artigos em 3 categorias: A (quando o estudo preenche mais de $80 \%$ dos critérios estabelecidos no STROBE), B (quando 50-80\% dos critérios do STROBE estão sendo alcançados), C (quando menos de $50 \%$ dos critérios estão preenchidos).

\section{RESULTADOS}

$\mathrm{Na}$ busca inicial, foram identificadas 1669 publicações. Foram filtrados os estudos entre 2014 e outubro de 2019, restando 605. Após análise dos títulos, 43 estudos foram selecionados, além de cinco estudos provenientes da lista de referências de outras publicações. Dentre esses, um estudo duplicado foi excluído. Após leitura dos resumos, 20 estudos foram considerados compatíveis, sendo, então, selecionados para análise do texto completo. Nesta etapa, foram excluídos quatro estudos por não especificarem os resultados de PE grave (Figura 1). 
Figura 1 - Esquematização do processo de seleção dos artigos.

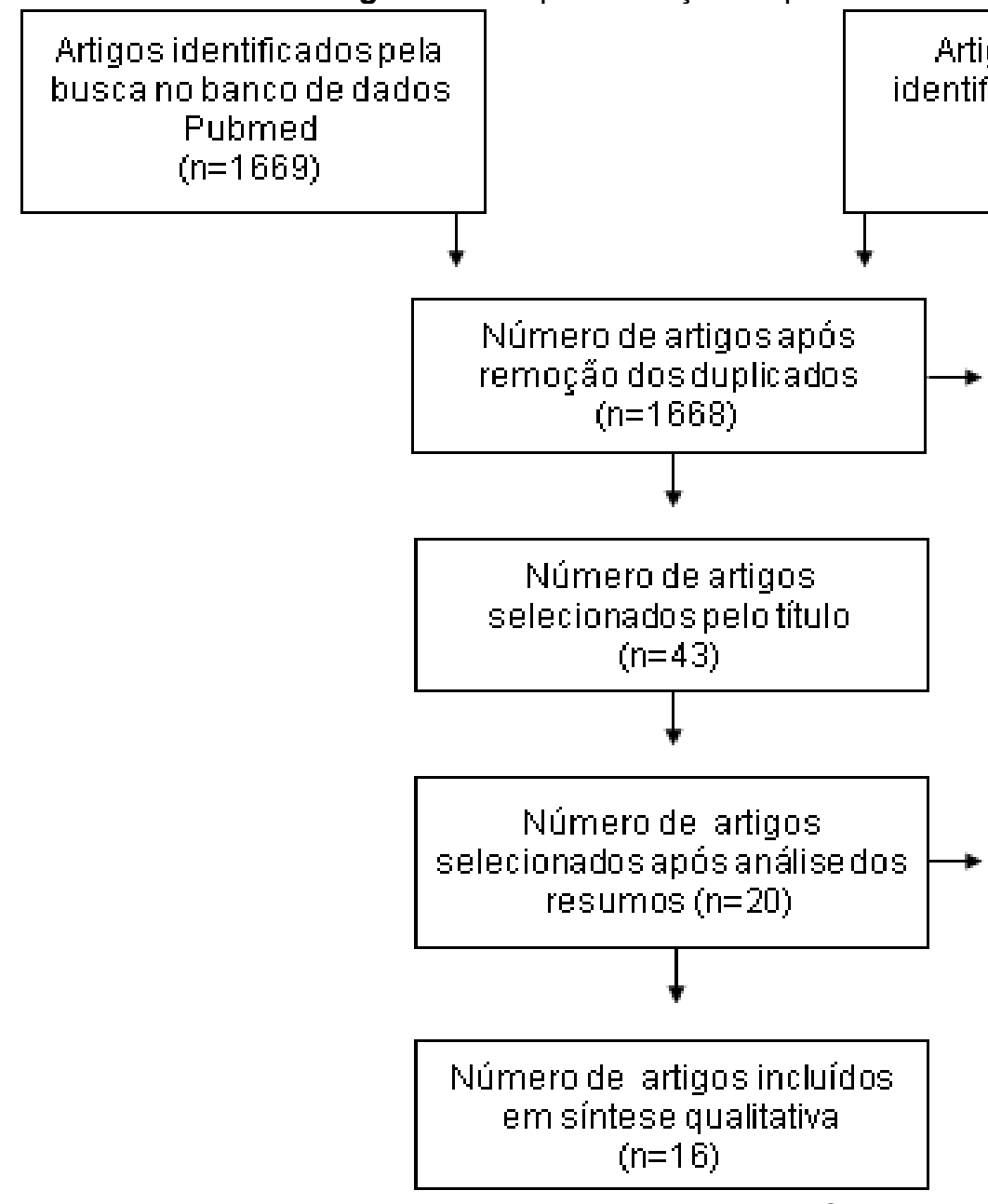

Artigos adicionais

identificados por outras

fontes

$(n=5)$
Número de artigos excluídos

Artigos anteriores a 2014 $(n=1063)$

Fonte: Almeida WS, et al., 2020.

Dos 16 artigos selecionados, 2 (12,5\%) foram desenvolvidos com populações da América do Sul; 2 (12,5\%), da América do Norte; 6 (37,5\%) da África; 3 (18,75\%) da Ásia e $3(18,75 \%)$ da Europa.

A amostra dos estudos incluídos variou entre 50 e 25391 gestantes, sendo estes classificados como estudos transversais, de coorte, retrospectivos e caso-controle. A porcentagem de casos de PE grave variou de $0,8 \%$ até $100 \%$.

A mortalidade neonatal foi avaliada nos 16 estudos e a maior porcentagem identificada foi de $81 \%$, enquanto a menor foi de $0 \%$, sendo a mediana das proporções de mortalidade igual a $9,32 \%$. No que diz respeito aos demais resultados neonatais, a prematuridade foi avaliada em $5(31,25 \%)$ estudos, sendo que a mediana de RNs prematuros foi de 33,9\%. Já o score de APGAR foi analisado em 7 (43,75\%) artigos. O peso ao nascer, em 9 (56,25\%), sendo a mediana de peso 2042,65g, classificando esses RNs como possuidores de baixo peso ao nascer. O número de internações em UTI, foi avaliado em 7 (43,75\%) estudos e a mediana da porcentagem dos RNs internados foi de $46,6 \%$. Nem todos os artigos expuseram dados acerca de resultados neonatais, sendo que $14(87,5 \%)$ discorrem sobre peso ao nascer, escore de APGAR, internações em CTI e prematuridade.

Por outro lado, a mortalidade materna não foi discutida na maioria dos artigos avaliados, sendo que apenas sete deles $(43,75 \%)$ discorreram sobre esse parâmetro. Nestes, a mortalidade variou de $0 \%$ a $4,4 \%$ das amostras, sendo a mediana das porcentagens de $1 \%$. Comparando-se a mortalidade materna com a mortalidade neonatal, percebe-se que, em todos artigos nos quais a mortalidade materna foi avaliada, houve mortalidade neonatal associada à mortalidade materna (Quadro 1). 
Quadro 1 - Identificação dos artigos selecionados e descrição dos resultados.

\begin{tabular}{|c|c|c|c|c|c|c|c|}
\hline & TítULo & $\begin{array}{c}\text { ANO DE } \\
\text { PUBLICAÇÃO } \\
\end{array}$ & AUTOR & TIPO DE ESTUDO & $\begin{array}{l}\text { MORTALIDADE } \\
\text { MATERNA }\end{array}$ & MORTALIDADE NEONATAL & RESULTADOS NEONATAIS \\
\hline 1 & $\begin{array}{c}\text { Maternal, fetal and neonatal } \\
\text { outcome among different types of } \\
\text { hipertensive disorders associating } \\
\text { pregnancy needing intensive care } \\
\text { management }\end{array}$ & 2018 & Ahmed M Maged & Caso-controle & $9(1.9 \%)$ & $\begin{array}{c}\text { Mortalidade perinatal: } 50 \\
(10,6 \%)\end{array}$ & 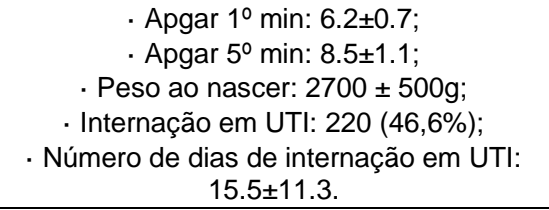 \\
\hline 2 & $\begin{array}{c}\text { Can thrombophilia worsen maternal } \\
\text { and perinatal outcomes in cases of } \\
\text { severe preeclampsia? }\end{array}$ & 2017 & Baptista FS & Coorte retrospectiva & Não consta & $\begin{array}{c}\text { - Mortalidade intrauterina: } 12 / 97 \\
(12,4 \%) ; \\
\text { - Mortalidade neonatal precoce: } \\
8 / 72(11,1 \%) ; \\
\text { - Mortalidade neonatal tardia: } \\
4 / 72(5.6 \%) \\
\text { - Mortalidade perinatal: } 25 / 85 \\
(29.4 \%) . \\
\end{array}$ & $\begin{array}{l}\text { - Apgar < } 7 \text { no } 1 \text { min: } 33 / 85(38,8 \%) \text {; } \\
\text { - Apgar < } 7 \text { no } 5 \text { min: } 7 / 85(8.2 \%) \text {; } \\
\text {. Peso ao nascer: } 1262 \pm 115.27 \mathrm{~g} ; \\
\text { - Internação em UTI: } 52 / 71(73.2 \%) \text {. }\end{array}$ \\
\hline 3 & $\begin{array}{c}\text { Characteristics and outcomes of } \\
\text { pacients with eclampsia and severe } \\
\text { pre-eclampsia in a rural hospital in } \\
\text { Western Tanzania: a retrospective } \\
\text { medical record study }\end{array}$ & 2015 & Rob Mooji & $\begin{array}{l}\text { Estudo retrospectivo } \\
\text { (análise de prontuários) }\end{array}$ & Não consta & Mortalidade perinatal: $6(27 \%)$ & $\begin{array}{c}\text { Peso ao nascer: } 2600 \pm 800 \mathrm{~g} ; \\
\text {. Baixo peso ao nascer }(<2.500 \mathrm{~g}): 11 \\
(37 \%) ; \\
\text { - Muito-baixo peso ao nascer }(<1.500 \mathrm{~g}): 2 \\
(7 \%) \text {. }\end{array}$ \\
\hline 4 & $\begin{array}{c}\text { Criteria-based audit of quality of care } \\
\text { to women with severe pre-eclampsia } \\
\text { and eclampsia in a referral hospital } \\
\text { in Accra, Ghana }\end{array}$ & 2015 & Joyce L. Browne & $\begin{array}{l}\text { Entrevista } \\
\text { semiestruturada/Estudo } \\
\text { transversal }\end{array}$ & Não consta & $\begin{array}{c}\text { Mortalidade perinatal: } 2(4.0 \% \\
\text { do total) }\end{array}$ & Não consta \\
\hline 5 & $\begin{array}{l}\text { Double-notches: association of } \\
\text { uterine artery notch forms with } \\
\text { pregnancy outcome and severity of } \\
\text { preeclampsia }\end{array}$ & 2015 & Ibrahim Polat & $\begin{array}{l}\text { Estudo retrospectivo } \\
\text { (análise de prontuários) }\end{array}$ & Não consta & $16(6.0 \%)$ & $\begin{array}{c}\text { Peso ao nascer: } 1407.9 \pm 521.3 ; \\
\text { Apgar 10 minuto: } 6.1 \pm 1.8 ; \\
\text { Apgar } 5^{\circ} \text { minuto: } 8.0 \pm 1.6 \\
\text {. Internação em UTI Neonatal: } 235 \text { (88.3\%). }\end{array}$ \\
\hline 6 & $\begin{array}{l}\text { Maternal and fetal outcome in pre- } \\
\text { eclampsia in a secondary care } \\
\text { hospital in South India }\end{array}$ & 2015 & $\begin{array}{l}\text { Parveen M } \\
\text { Aabidha }\end{array}$ & Coorte prospectiva & 0 & Mortalidade perinatal: $4.3 \%$ & $\begin{array}{c}\text { Prematuridade: } 23.65 \% \\
\text { - Baixo peso ao nascer: } 7.52 \% \text {. }\end{array}$ \\
\hline 7 & $\begin{array}{l}\text { Maternal and neonatal outcomes in } \\
\text { women with severe early onset pre- } \\
\text { eclampsia before } 26 \text { weeks of } \\
\text { gestation, a case series }\end{array}$ & 2017 & MF van Ostwaad & Série de casos & 0 & $\begin{array}{l}\text { Morte neonatal } \leq 7 \mathrm{~d}: 13(8,5 \%) ; \\
\text { - Morte neonatal } \geq 7 \mathrm{~d}: 10(7,9 \%) ; \\
\text { - Morte perinatal: } 103(74 \%) ; \\
\text { - Mortalidade total: } 113(81 \%)\end{array}$ & $\begin{array}{l}\text { - Baixo peso ao nascer: } 127 ; \\
\text { Pequeno para a idade gestacional: } 80 \\
(27 \%) ; \\
\text {. Ressuscitação neonatal: } 50(36 \%) \text {. }\end{array}$ \\
\hline 8 & $\begin{array}{c}\text { Maternal clinical disease } \\
\text { characteristics and maternal and } \\
\text { neonatal outcomes in twin and } \\
\text { singleton pregnancies with severe } \\
\text { preeclampsia }\end{array}$ & 2015 & $\begin{array}{l}\text { Katherine A } \\
\text { Connolly }\end{array}$ & Coorte retrospectiva & Não consta & $8(1,7 \%)$ & $\begin{array}{l}\text { Pequeno para a idade gestacional: } 84 \\
\text { (18\%); } \\
\text { - Ventilação assistida: } 55 \text { (11,8\%); } \\
\text { - Admissão em UTI: } 208 \text { (44,7\%). }\end{array}$ \\
\hline
\end{tabular}




\begin{tabular}{|c|c|c|c|c|c|c|c|}
\hline & TítULO & $\begin{array}{c}\text { ANO DE } \\
\text { PUBLICAÇÃO }\end{array}$ & AUTOR & TIPO DE ESTUDO & $\begin{array}{l}\text { MORTALIDADE } \\
\text { MATERNA }\end{array}$ & MORTALIDADE NEONATAL & RESULTADOS NEONATAIS \\
\hline 9 & $\begin{array}{l}\text { Perinatal outcome in singleton } \\
\text { pregnancies complicated with } \\
\text { preeclampsia and eclampsia in } \\
\text { Ecuador }\end{array}$ & 2016 & KYN Phoa & Estudo transversal & Não consta & $17(13,7 \%)$ & $\begin{array}{c}\text { - Prematuridade: } 42(33,9 \%) ; \\
\text { Pequeno para a idade gestacional: } 55 \\
\text { (44,3\%); } \\
\text { - Baixo peso ao nascer: } 65(52,4 \%) ; \\
\text { - Admissão em UTI ou cuidado } \\
\text { intermediário: } 42(33,9 \%) ; \\
\text { - Score de Apgar no } 5^{\circ} \text { minuto } \leq 7: 15 \\
(12 \%) .\end{array}$ \\
\hline 10 & $\begin{array}{l}\text { Placental histopathological lesions in } \\
\text { correlation with neonatal outcome in } \\
\text { preeclampsia with and without } \\
\text { severe features }\end{array}$ & 2018 & Eran Weiner & Coorte retrospectiva & Não consta & $7(2,5 \%)$ & $\begin{array}{c}\text { - Pequeno para a idade gestacional: } 74 \\
(26,1 \%) ; \\
\text {. Internação em UTI: } 130(45,8 \%) ; \\
\text {. Escore de Apgar } \leq 7 \text { no } 5^{\circ} \text { minuto: } 12 \\
\text { (4,2\%); } \\
\text {. Sepse neonatal: } 4 \text { (1,4\%). }\end{array}$ \\
\hline 11 & $\begin{array}{l}\text { Point of care ultrasound: does the } \\
\text { presence of ascites in severe pre- } \\
\text { eclampsia correlate with poor } \\
\text { maternal and neonatal outcome? }\end{array}$ & 2019 & $\begin{array}{l}\text { Celestin } \\
\text { Mbonyizina }\end{array}$ & Coorte prospectiva & $5(4,4 \%)$ & $9(9,1 \%)$ & $\begin{array}{c}\text { - Internação em UTI: } 40(87,2 \%) \text { com ascite, } \\
35 \text { (68\%) sem ascite; } \\
\text { · Apgar no 50 min } 7,5 \pm 0.23 \text { com ascite, } 8 \pm \\
0.25 \text { sem ascite; } \\
\text { - Peso ao nascer } 1587.3 \pm 77.03 \text { com } \\
\text { ascite, } 2011.6 \pm 103.5 \text { sem ascite. }\end{array}$ \\
\hline 12 & $\begin{array}{l}\text { Severe preeclampsia and } \\
\text { eclampsia: incidence, complications, } \\
\text { and perinatal outcomes at a low- } \\
\text { resouce setting, Mpilo Central } \\
\text { Hospital, Bulawayo, Zimbabwe }\end{array}$ & 2017 & $\begin{array}{l}\text { Solwayo } \\
\text { Ngwenya }\end{array}$ & Coorte retrospectiva & $1(1 \%)$ & $44(46,3 \%)$ & $\begin{array}{c}\text {. Apgar baixo: } 5(5,2 \%) \\
\text {. Prematuridade: } 30 \text { (31,5\%). }\end{array}$ \\
\hline 13 & $\begin{array}{c}\text { Severe preeclampsia versus HELLP } \\
\text { Syndrome: Maternal and Perinatal } \\
\text { Outcomes at }<34 \text { and } \geq 34 \text { weeks } \\
\text { gestation }\end{array}$ & 2015 & Tugba Kinay & Estudo transversal & Não consta & $\begin{array}{c}\cdot<34 \text { semanas: } 12(14 \%) \\
\text { natimortos; } \\
\cdot>34 \text { semanas: } 3(2,7 \%) \\
\text { natimortos }\end{array}$ & $\begin{array}{c}\diamond<34 \text { semanas: } \\
\text {. Prematuridade: } 86(100 \%) ; \\
\text {. Apgar baixo: } 44(51,2 \%) ; \\
\text {. Baixo peso: } 85(98,8 \%) \text {. } \\
\diamond>34 \text { semanas: } \\
\text {. prematuridade: } 49(44,1 \%) ; \\
\text {. Apgar baixo: } 2(7,7 \%) ; \\
\text {. Baixo peso: } 12(46,2 \%) . \\
\end{array}$ \\
\hline 14 & $\begin{array}{c}\text { The feto-maternal Outcome of } \\
\text { preeclampsia with severe feature } \\
\text { and eclampsia in Abakaliki, South- } \\
\text { East Nigeria }\end{array}$ & 2016 & $\begin{array}{l}\text { Leonard Ogbonna } \\
\text { Ajah }\end{array}$ & Caso-controle & $2(1 \%)$ & $8(2,3 \%)$ & Não consta \\
\hline 15 & $\begin{array}{c}\text { The placental component and } \\
\text { obstetric outcome in severe } \\
\text { preeclampsia with ans without } \\
\text { HELLP syndrome }\end{array}$ & 2016 & Eran Weiner & $\begin{array}{l}\text { Estudo retrospectivo } \\
\text { (análise de prontuários) }\end{array}$ & Não consta & 0 & $\begin{array}{l}\text {. Peso ao nascer: } 2498 \pm 436 \\
\text { - Internação em UTI: } 151 \text { (62,1\%); } \\
\text { · Apgar } \leq 7 \text { no } 5^{\circ} \text { min: } 3(1,2 \%)\end{array}$ \\
\hline 16 & $\begin{array}{c}\text { Resultados maternos y perinatales } \\
\text { del tratamiento expectante de la } \\
\text { preeclampsia severa }\end{array}$ & 2018 & $\begin{array}{l}\text { Juan Gustavo } \\
\text { Vásquez- } \\
\text { Rodriguez }\end{array}$ & $\begin{array}{l}\text { Estudo observacional } \\
\text { retrospectivo } \\
\text { (análise de prontuários) }\end{array}$ & $0,00 \%$ & $10(24,39 \%)$ & $\begin{array}{c}\text {. Prematuridade: } 23(100 \%) \\
\text {. Internação em UTI: } 16(39,02 \%) \\
\text {. CIUR: } 9(21,95 \%) .\end{array}$ \\
\hline
\end{tabular}

Legenda: UTI = unidade de terapia intensiva. CIUR = crescimento intrauterino restrito.

Fonte: Almeida WS, et al., 2020. 
Quanto à qualidade dos artigos selecionados, todos apresentam boa qualidade quanto ao contexto e justificativa introdutórios, desenho de estudo, contexto metodológico (local, data, quantidade de participantes), descrição dos principais resultados, discussão e interpretação dos resultados e validade externa. Já, quanto ao título, resumo e objetivos, 15 artigos apresentaram boa qualidade. Avaliando-se critérios de elegibilidade dos participantes, fonte dos dados, resultados quanto ao agrupamento e descrição dos participantes e outras análises específicas de cada tipo de estudo, 14 artigos foram classificados como bons. Quanto às variáveis, ao tamanho do estudo, aos métodos estatísticos utilizados e às limitações, 13 artigos são de boa qualidade. Já, considerando-se os vieses, 12 artigos descreveram medidas adotadas para redução de tais erros. Onze artigos apresentaram descrição favorável quando às variáveis quantitativas. Quanto ao financiamento, apenas 5 artigos descreveram as respectivas fontes financiadoras. Já quanto aos desfechos, em 11 artigos tal critério não se aplica e apenas 1 dos demais artigos não apresentou boa qualidade quanto ao referido critério (Tabela 1).

Tabela 1 - Avaliação da qualidade dos artigos de acordo com o checklist STROBE.

\begin{tabular}{|c|c|c|c|c|c|c|c|c|c|c|c|c|c|c|c|c|c|c|c|c|c|c|c|}
\hline $\begin{array}{c}\text { Itens } \\
\text { STROBE }\end{array}$ & $\mathbf{1}$ & $\mathbf{2}$ & $\mathbf{3}$ & $\mathbf{4}$ & $\mathbf{5}$ & $\mathbf{6}$ & $\mathbf{7}$ & $\mathbf{8}$ & $\mathbf{9}$ & $\mathbf{1 0}$ & $\mathbf{1 1}$ & $\mathbf{1 2}$ & $\mathbf{1 3}$ & $\mathbf{1 4}$ & $\mathbf{1 5}$ & $\mathbf{1 6}$ & $\mathbf{1 7}$ & $\mathbf{1 8}$ & $\mathbf{1 9}$ & $\mathbf{2 0}$ & $\mathbf{2 1}$ & $\mathbf{2 2}$ & Total \\
\hline Artigo & \multicolumn{10}{|c|}{$\mathbf{9}$} \\
\hline $\mathbf{1}$ & 1 & 1 & 1 & 1 & 1 & 1 & 1 & 1 & 1 & 1 & 1 & 1 & 0 & 0 & NA & 1 & 0 & 1 & 1 & 1 & 1 & 0 & 17 \\
\hline $\mathbf{2}$ & 1 & 1 & 1 & 1 & 1 & 1 & 0 & 1 & 1 & 0 & 1 & 1 & 1 & 1 & 1 & 0 & 1 & 1 & 1 & 1 & 1 & 0 & 18 \\
\hline $\mathbf{3}$ & 1 & 1 & 1 & 1 & 1 & 1 & 1 & 1 & 0 & 1 & 0 & 0 & 1 & 1 & NA & 1 & 1 & 1 & 1 & 1 & 1 & 0 & 17 \\
\hline $\mathbf{4}$ & 1 & 1 & 1 & 1 & 1 & 1 & 1 & 1 & 1 & 1 & 1 & 1 & 1 & 0 & NA & 1 & 1 & 1 & 1 & 1 & 1 & 1 & 20 \\
\hline $\mathbf{5}$ & 0 & 1 & 1 & 1 & 1 & 1 & 1 & 1 & 1 & 1 & 1 & 1 & 1 & 1 & NA & 1 & 1 & 1 & 1 & 1 & 1 & 0 & 19 \\
\hline $\mathbf{6}$ & 1 & 1 & 1 & 1 & 1 & 1 & 0 & 1 & 0 & 0 & 0 & 0 & 1 & 1 & NA & 1 & 1 & 1 & 1 & 1 & 1 & 0 & 15 \\
\hline $\mathbf{7}$ & 1 & 1 & 1 & 1 & 1 & 1 & 1 & 1 & 1 & 1 & 1 & 1 & 1 & 1 & NA & 1 & 1 & 1 & 1 & 1 & 1 & 1 & 21 \\
\hline $\mathbf{8}$ & 1 & 1 & 1 & 1 & 1 & 1 & 1 & 1 & 0 & 1 & 1 & 1 & 1 & 1 & 0 & 0 & 1 & 1 & 1 & 1 & 1 & 0 & 18 \\
\hline $\mathbf{9}$ & 1 & 1 & 1 & 1 & 1 & 1 & 1 & 1 & 1 & 1 & 1 & 1 & 0 & 1 & NA & 1 & 0 & 1 & 1 & 1 & 1 & 0 & 18 \\
\hline $\mathbf{1 0}$ & 1 & 1 & 1 & 1 & 1 & 1 & 1 & 1 & 1 & 1 & 1 & 1 & 1 & 1 & 1 & 1 & 1 & 1 & 1 & 1 & 1 & 0 & 21 \\
\hline $\mathbf{1 1}$ & 1 & 1 & 1 & 1 & 1 & 1 & 1 & 1 & 1 & 1 & 1 & 0 & 1 & 1 & 1 & 1 & 1 & 1 & 1 & 1 & 1 & 0 & 20 \\
\hline $\mathbf{1 2}$ & 1 & 1 & 0 & 1 & 1 & 1 & 0 & 0 & 1 & 1 & 0 & 1 & 1 & 1 & 1 & 1 & 1 & 1 & 0 & 1 & 1 & 0 & 16 \\
\hline $\mathbf{1 3}$ & 1 & 1 & 1 & 1 & 1 & 0 & 1 & 1 & 0 & 0 & 1 & 1 & 1 & 1 & NA & 1 & 1 & 1 & 0 & 1 & 1 & 1 & 17 \\
\hline $\mathbf{1 4}$ & 1 & 1 & 1 & 1 & 1 & 0 & 1 & 0 & 1 & 1 & 0 & 1 & 1 & 1 & NA & 1 & 1 & 1 & 1 & 1 & 1 & 1 & 18 \\
\hline $\mathbf{1 5}$ & 1 & 1 & 1 & 1 & 1 & 1 & 1 & 1 & 1 & 1 & 1 & 1 & 1 & 1 & NA & 1 & 0 & 1 & 1 & 1 & 1 & 1 & 20 \\
\hline $\mathbf{1 6}$ & 1 & 1 & 1 & 1 & 1 & 1 & 1 & 1 & 1 & 1 & 0 & 1 & 1 & 1 & NA & 1 & 1 & 1 & 0 & 1 & 1 & 0 & 18 \\
\hline Total & 15 & 1 & 15 & 16 & 16 & 14 & 13 & 14 & 12 & 13 & 11 & 13 & 14 & 14 & 4 & 14 & 13 & 16 & 13 & 16 & 16 & 5 \\
\hline
\end{tabular}

Legenda: Os artigos encontram-se enumerados na primeira coluna à esquerda de acordo com a numeração atribuída a eles na tabela 1 . Na coluna à direita encontra-se a pontuação total obtida por cada artigo no STROBE. A última linha corresponde ao número de artigos que pontuou em cada um dos 22 itens do STROBE. NA = não se aplica. Fonte: Almeida WS, et al., 2020.

De acordo com os critérios de Mataratzis PSR, et al (2010), 68,5\% dos artigos da presente revisão foram classificados como A, tornando-os fontes confiáveis (Tabela 2).

Tabela 2 - Classificação da qualidade dos artigos.

\begin{tabular}{|c|c|c|c|}
\hline Artigo & STROBE & Porcentagem & Classificação \\
\hline $\mathbf{1}$ & 17 & 77,3 & $\mathrm{~B}$ \\
\hline $\mathbf{2}$ & 18 & 81,8 & $\mathrm{~A}$ \\
\hline $\mathbf{3}$ & 17 & 77,3 & $\mathrm{~B}$ \\
\hline $\mathbf{4}$ & 20 & 90,9 & $\mathrm{~A}$ \\
\hline $\mathbf{5}$ & 19 & 86,4 & $\mathrm{~A}$ \\
\hline $\mathbf{6}$ & 15 & 68,2 & $\mathrm{~B}$ \\
\hline $\mathbf{7}$ & 21 & 95,5 & $\mathrm{~A}$ \\
\hline $\mathbf{8}$ & 18 & 81,8 & $\mathrm{~A}$ \\
\hline $\mathbf{9}$ & 18 & 81,8 & $\mathrm{~A}$ \\
\hline $\mathbf{1 0}$ & 21 & 95,5 & $\mathrm{~A}$ \\
\hline $\mathbf{1 1}$ & 20 & 90,9 & $\mathrm{~A}$ \\
\hline $\mathbf{1 2}$ & 16 & 72,7 & $\mathrm{~B}$ \\
\hline $\mathbf{1 3}$ & 17 & 77,3 & $\mathrm{~B}$ \\
\hline $\mathbf{1 4}$ & 18 & 81,8 & $\mathrm{~A}$ \\
\hline $\mathbf{1 5}$ & 20 & 90,9 & $\mathrm{~A}$ \\
\hline $\mathbf{1 6}$ & 18 & 81,8 & $\mathrm{~A}$ \\
\hline
\end{tabular}

Fonte: Almeida WS, et al., 2020. 


\section{DISCUSSÃO}

Os resultados obtidos, de modo geral, suportam a existência de um impacto do quadro de PE grave nos diversos desdobramentos à morbimortalidade materno-infantil, ainda que em abordagens e proporções distintas. O grau de consenso da comunidade acadêmica sobre o tema deve, portanto, considerar as particularidades dos países de origem de cada um dos estudos selecionados.

Por adotar os critérios diagnósticos para PE grave sugeridos pelo ACOG (WISNER K, 2019), a padronização da classificação representa um ponto positivo que permite a comparação dos resultados, minimizando possíveis vieses de seleção das amostras. Apenas o estudo de Ajah LO, et al. (2016) não especifica os critérios adotados na classificação da PE grave por trabalhar com dados secundários constantes nos prontuários.

Dentre os critérios utilizados para inclusão de gestantes nas amostras consideradas como PE grave estão as lesões de órgãos-alvo, os sinais e sintomas de comprometimento sistêmico e a morbidade fetal, mesmo na ausência de proteinúria. Estes critérios mantêm consonância com aqueles adotados pelo ACOG e podem estar relacionados a piores resultados maternos e neonatais (DONG X, et al., 2017). Diferentemente, a dosagem de proteinúria por fita reagente está contraindicada, exceto se não houver outros métodos quantitativos disponíveis (WISNER K, 2019). Os estudos de Cherian A, et al. (2015) e Ngwenya S, et al. (2017) não especificam esta medida.

Na revisão sistemática realizada por Abalos E, et al. (2013), que analisou estudos publicados entre os anos de 2002 e 2010, a incidência global de PE encontrada variou entre 1,2\% e 4,2\%. Entretanto, esse estudo não estratificou a incidência das formas leve e grave e ainda salientou que a falta de dados dificulta uma análise mais fidedigna.

A obtenção de estimativas acuradas da incidência de PE, sobretudo na forma grave que é o foco dessa revisão, é dificultada devido à falta de padronização dos critérios diagnósticos nas bases de dados populacionais (HUTCHEON JA, et al., 2011). Segundo estudo de Kongwattanakul K, et al. (2018), realizado na Tailândia entre 2012 e 2016, a incidência de PE grave é de aproximadamente $0,8 \%$ de todas as gestações. Nos artigos selecionados nessa revisão a incidência de PE grave variou de $0,8 \%$ a $3,3 \%$ de todas as gestações. Por outro lado, 11 dos estudos analisados não apresentaram essa análise, uma vez que possuem como amostra total pacientes já diagnosticadas com PE leve ou grave.

Variações da incidência da PE grave são justificadas por diferentes características populacionais, como distribuição da idade, e diagnóstico (HUTCHEON JA, et al., 2011). Ainda assim, a heterogeneidade das regiões e das características populacionais e a ausência da análise da incidência da PE grave em parte dos estudos sugerem que a incidência da PE grave apresentada nessa revisão seja analisada com cautela.

Segundo as definições da OMS, há uma distinção entre os parâmetros de mortalidade neonatal e perinatal adotados pelos estudos (OMS, 2019; OMS, 2011). Na presente revisão, detectamos uma variação de $4 \%$ a $74 \%$, para mortalidade perinatal e $2,3 \%$ a $27,6 \%$, para mortalidade neonatal nos estudos que abordaram essas variáveis (BROWNE JL, et al., 2015; OSTWAAD MFV, et al., 2017; AJAH LO, et al., 2016; NGWENYA S, et al., 2017). Esses dados vão ao encontro da literatura vigente, visto que é conhecida a maior incidência de mortalidade perinatal em conceptos de gestantes com PE grave quando comparada àquela dos RN de mães normotensas (OLIVEIRA CA, 2006).

Pode-se depreender uma importante relação entre as características socioeconômicas e as taxas de mortalidade perinatal e neonatal. Segundo Duley L, et al. (2009), um quarto do número de mortes neonatais e de natimortos em países em desenvolvimento está associado a PE e eclâmpsia. De acordo com esse estudo, nesses países, muitos hospitais públicos possuem menor acesso ao cuidado intensivo neonatal, portanto a morbimortalidade associada à $\mathrm{PE}$ e à eclâmpsia é considerada maior do que em locais onde esse acesso é facilitado (DULEY L, 2009). 
Esta revisão encontrou resultados consonantes a esses no estudo de Phoa KYN, et al. (2016), que descreveu os resultados perinatais em gestantes equatorianas de baixa renda com PE e eclâmpsia e observou piores desfechos neonatais, sendo eles APGAR baixo, baixo peso ao nascer (BPN), PIG e prematuridade, em gestações com PE grave que não tiveram acompanhamento pré-natal oportuno.

Observou-se também uma ampla variabilidade de resultados neonatais adversos. Entretanto, sendo os mais frequentes e os mais relevantes, foram abordados como os principais resultados o escore de APGAR baixo, BPN, prematuridade e necessidade de internação em UTI neonatal. Os escores de APGAR, o peso ao nascer e a idade gestacional são altamente associados à sobrevivência e, em combinação, compreendem uma medida do bem-estar do RN, do sucesso da reanimação, do tamanho e da maturidade do RN. A mediana das proporções de prematuridade, necessidade de internação em UTI e mediana de peso encontradas, respectivamente, $33,9 \%, 46,6 \%$ e $2042,65 \mathrm{~g}$ são justificáveis pela maior severidade da PE grave (FEBRASGO, 2017).

O estudo de Polat I, et al. (2015), que comparou resultados neonatais de grupos com PE leve e PE grave, concluiu que houve maior necessidade de internação em UTI neonatal no grupo com PE grave, chegando a mais de $88 \%$ dos neonatos, com diferença estatisticamente significativa. Outros estudos também ressaltam a substancial necessidade de cuidados intensivos para neonatos nesse contexto, que muitas vezes aparecem como mais da metade da população neonatal estudada (NGWENYA S, et al., 2017; VÁZQUEZ-RODRIGUEZ JG, et al., 2018).

Houve, ainda, associação entre o escore de APGAR baixo nos primeiros cinco minutos em neonatos de gestantes com PE grave, quando comparados aos resultados de gestantes com PE leve (POLAT I, et al., 2015). Esses achados são similares aos de outro estudo analisado (PHOA KYN, et al., 2016). Entretanto, uma análise estatística dos dados de escore de APGAR baixo não foi realizada nessa revisão pois os artigos apresentam tais números de forma variável.

Nos estudos que mostraram um escore de APGAR $\leq 7$ no quinto minuto houve uma variação de 1,2 a $51,2 \%$ dos RNs. Já nos estudos que mostraram uma média de escore APGAR o dado variou entre 7,5 a 8,5. Um estudo mostrou escore de APGAR $\leq 7$ no primeiro minuto em $38,8 \%$ dos RNs e outros 2 estudos mostraram uma média de escore de APGAR no primeiro minuto de 6,1 e 6,2.

Os resultados da presente pesquisa mostram que o BPN está associado a mortalidade neonatal em consonância com o estudo retrospectivo de prontuários de gestantes com PE grave ou eclâmpsia em um hospital na Tanzânia realizado por Mooij R, et al. (2015) que menciona o BPN como preditor de morte fetal e neonatal. Esses resultados corroboram a literatura atual, uma vez que, de acordo com a Organização Mundial da Saúde (OMS), a relação entre BPN e mortalidade neonatal é bem estabelecida, podendo chegar a 60 a 80\% dos óbitos nessa faixa etária (OMS, 2018).

A prematuridade consiste em um importante fator de risco para alterações no desenvolvimento neurológico, respiratório, complicações oftalmológicas e gastrointestinais, bem como dificuldade respiratória, apneia, icterícia, kernicterus, dificuldades de nutrição, hipoglicemia, convulsões e hospitalização prolongada (OMS, 2011).

Em países desenvolvidos, corresponde à maior causa de morbimortalidade neonatal, sendo um desfecho frequente em gestações que cursam com PE grave (RI G, et al., 2008; DULEY L, 2009). Em consonância com esses dados, os estudos de Cherian A, et al. (2015), de Kinay T, et al. (2015) e de Vázquez-Rodriguez JG, et al. (2018), analisados na presente revisão, apontam a prematuridade como a complicação neonatal mais comum encontrada nas gestantes com PE avaliadas, registrando $23,65 \%$, $44,1 \%$ e $100 \%$, respectivamente.

A publicação de Phoa KYN, et al. (2016), por sua vez, também coloca o nascimento pré-termo como um resultado da PE, porém menos prevalente que o BPN. Sob uma perspectiva diferente, o trabalho de 
Ngwenya S, et al. (2017) demonstra que a maioria (81,5\%) das gestantes avaliadas foram admitidas no serviço devido a uma combinação entre a prematuridade e outros fatores, como BPN e síndrome de estresse respiratório.

A mortalidade materna no contexto da PE grave foi um assunto pouco explorado nos estudos analisados, sendo citada em sete deles (MAGED AM, et al., 2018; CHERIAN A, et al., 2015; OSTWAAD MFV, et al., 2017; MBONYIZINA C, et al., 2019; NGWENYA S, et al., 2017; ANANTH CV, et al., 2016; AJAH LO, et al., 2016; VÁSQUEZ-RODRIGUEZ JG, et al., 2018). Nestes, esse parâmetro variou de 0 a $4,4 \%$. Esse achado difere da literatura vigente, que aponta que 10 a $15 \%$ das mortes maternas estão associadas à PE (OMS, 2011). No entanto, essa porcentagem se refere à mortalidade geral por PE, sem estratificar a gravidade.

Dados sobre mortalidade materna correlacionada especificamente à PE grave são escassos na literatura. Uma outra hipótese para justificar as taxas inferiores de mortalidade encontradas neste estudo em relação às existentes na literatura, é que a maioria dos estudos abordados foi executada em centros terciários de saúde, que contam com melhores recursos de assistência obstétrica, o que pode ter impactado positivamente nos resultados.

Sobre as características socioeconômicas e seu impacto à saúde, é sabido que mulheres que vivem em países com baixas condições socioeconômicas apresentam maior risco de desenvolver PE quando comparadas àquelas residentes em países desenvolvidos (POON LC, et al., 2019).

Os estudos selecionados compõem um grupo heterogêneo, abrangendo desde o contexto de países subdesenvolvidos, com baixas condições socioeconômicas, que refletem diretamente na qualidade de assistência à saúde e na disponibilidade de pré-natal apropriado, como também a realidade de países desenvolvidos, que sustentam bons parâmetros de cuidados em saúde e de assistência pré-natal. Apesar dessa discrepância, optou-se por analisar os estudos coletivamente, em detrimento da estratificação por continentes, devido ao pequeno número de publicações que preenchessem os critérios de inclusão estipulados. Consideramos, portanto, que a grande diferença na qualidade de assistência à saúde entre os estudos selecionados possa constituir uma limitação desta pesquisa.

Foram selecionados estudos oriundos de 14 países, com representantes dos cinco continentes, sendo que o continente com maior número de estudos encontrados foi a África. Esse padrão pode ser justificado pela maior prevalência de PE nos países africanos, conforme o estudo de Vata PK, et al. (2015), que aponta a prevalência de PE em $10 \%$ das gestações africanas, contra aproximadamente $2 \%$ das taxas globais. Segundo Wagnew M, et al. (2016), 56\% das mortes maternas de todo o mundo no ano de 2013 ocorreram na África Subsaariana, sendo que entre as principais causas estão as síndromes hipertensivas da gestação, predominantemente a PE e a eclâmpsia.

Diante disso, são necessários mais estudos sobre essa temática, para obter o aprimoramento dos dados estatísticos e, assim, orientar políticas de saúde específicas para as demandas de cada região, melhorando a assistência à saúde materno-neonatal.

\section{CONSIDERAÇÕES FINAIS}

Observou-se que a PE grave apresenta um impacto relevante na mortalidade neonatal e nos demais resultados neonatais avaliados: prematuridade, escore de APGAR baixo, baixo peso ao nascer e número de internações em UTI. Quanto à mortalidade materna, há uma lacuna de estudos no período analisado que explorem as correlações entre os óbitos das gestantes com PE grave. Por outro lado, percebe-se uma carência mundial de estudos sobre o tema. É fundamental estabelecer a identificação precoce dos sinais de gravidade da PE e oferecer o suporte adequado para minimizar os desfechos adversos observados no presente estudo. Reforça-se ainda que mais estudos sobre 0 assunto sejam realizados, gerando dados mais fidedignos, que traduzam o real panorama da PE grave e permitam que sejam criadas políticas de saúde oportunas para reduzir os danos causados por essa doença mundialmente. 


\section{REFERÊNCIAS}

1. ABALOS E, et al. Global and regional estimates of preeclampsia and eclampsia: A systematic review. European Journal of Obstetrics and Gynecology and Reproductive Biology, 2009; 170(1): 1-7.

2. AJAH LO, et al. The feto-maternal outcome of preeclampsia with severe features and eclampsia in Abakaliki, South-East Nigeria. Journal of Clinical and Diagnostic Research, 2016; 10(9): QC18-QC21.

3. ANANTH CV, et al. Serious maternal complications in relation to severe pre-eclampsia: a retrospective cohort study of the impact of hospital volume. BJOG: An International Journal of Obstetrics and Gynaecology, 2017; 124(8): 1246-1253.

4. BROWNE JL, et al. Criteria-based audit of quality of care to women with severe pre-eclampsia and eclampsia in a referral hospital in Accra, Ghana. PLoS ONE, 2015; 10(4): 1-12.

5. CHAIM SRP, et al. Hipertensão arterial na gestação e condições neonatais ao nascimento. ACTA Paulista de Enfermagem, 2008; 21(1): 53-58.

6. CHERIAN A, et al. Maternal and fetal outcome in pre-eclampsia in a secondary care hospital in South India. Journal of Family Medicine and Primary Care, 2015; 4(2): 257.

7. DE OLIVEIRA ACM, et al. Fatores maternos e resultados perinatais adversos em portadoras de pré-eclâmpsia em Maceió, Alagoas. Arquivos Brasileiros de Cardiologia, 2016; 106(2): 113-120.

8. DE OLIVEIRA CA, et al. Síndromes hipertensivas da gestação e repercussões perinatais. Revista Brasileira de Saude Materno Infantil, 2006; 6(1): 93-98.

9. DE OLIVEIRA TG, et al. Escore de Apgar e mortalidade neonatal em um hospital localizado na zona sul do município de São Paulo Apgar score and neonatal mortality in a hospital located in the southern area of São Paulo City, Brazil. einstein. São Paulo (SP), 2012; 10(111): 22-28.

10. DONG $X$, et al. Proteinuria in preeclampsia: Not essential to diagnosis but related to disease severity and fetal outcomes. Pregnancy Hypertension, 2017; 80(8): 60-64.

11. DULEY L. The Global Impact of Pre-eclampsia and Eclampsia. Seminars in Perinatology, 2009; 33(3): 130-137.

12. FEBRASGO. Pré-eclâmpsia nos seus diversos aspectos. Série Orientações e Recomendações FEBRASGO, $2017 ;$ [s.n.]: 56.

13. HUTCHEON JA, et al. Epidemiology of pre-eclampsia and the other hypertensive disorders of pregnancy. Best Practice and Research: Clinical Obstetrics and Gynaecology, 2011; 25(4): 391-403.

14. KAHHALE S, et al. Pré-eclampsia. Revista de Medicina, 2018; 97(2): 226.

15. KINAY T, et al. Severe preeclampsia versus HELLP syndrome: Maternal and perinatal outcomes at $<34$ and $\geq 34$ weeks' gestation. Balkan Medical Journal, 2015; 32(4): 359-363.

16. KONGWATTANAKUL $\mathrm{K}$, et al. Incidence, characteristics, maternal complications, and perinatal outcomes associated with preeclampsia with severe features and hellp syndrome. International Journal of Women's Health, 2018; 10[s.n.]: 371-377.

17. LOURENÇO NCV. Avaliação de citocinas pró e anti-inflamatórias e de fatores pró e anti-angiogênicos em placenta de gestantes com pré-eclâmpsia. Instituto de Biociências, Universidade Estadual Paulista, 2009; [s.n].

18. MAGED AM, et al. Maternal, fetal, and neonatal outcomes among different types of hypertensive disorders associating pregnancy needing intensive care management. Journal of Maternal-Fetal and Neonatal Medicine, 2018; [s.n.]: 7058.

19. MATARATZIS PSR, et al. Deficiências de micronutrientes em crianças e adolescentes com anemia falciforme: Uma revisão sistemática. Revista Brasileira de Hematologia e Hemoterapia, 2010; 32(3): 247-256.

20. MBONYIZINA C, et al. Point of care ultrasound: does the presence of ascites in severe pre-eclampsia correlate with poor maternal and neonatal outcome?. Tropical Medicine and International Health, 2019; 24(8): 1018-1022.

21. MOOIJ R, et al. Characteristics and outcomes of patients with eclampsia and severe pre-eclampsia in a rural hospital in Western Tanzania: A retrospective medical record study. BMC Pregnancy and Childbirth, 2015; 15(1): 1-7.

22. NGWENYA S. Severe preeclampsia and eclampsia: Incidence, complications, and perinatal outcomes at a low-resource setting, Mpilo central hospital, Bulawayo, Zimbabwe. International Journal of Women's Health, 2017; 9[s.n.]: 353-357.

23. PACHECO RIL, et al. Guidelines para publicação de estudos científicos. Parte 2 - Como publicar estudos observacionais. Revista Diagnóstico \& Tratamento, 2017; 22(3): 121-126.

24. PHOA KYN, et al. Perinatal outcome in singleton pregnancies complicated with preeclampsia and eclampsia in Ecuador. Journal of Obstetrics and Gynaecology, 2016; 36(5): 581-584.

25. POLAT I, et al. Double notches: Association of uterine artery notch forms with pregnancy outcome and severity of preeclampsia. Hypertension in Pregnancy, 2015; 34(1): 90-101.

26. POON LC, et al. The International Federation of Gynecology and Obstetrics (FIGO) initiative on pre-eclampsia: A pragmatic guide for first-trimester screening and prevention. International Journal of Gynecology and Obstetrics, 2019; 145(S1): 1-33.

27. RI G, et al. Epidemiology and causes of preterm birth. The Lancet, 2008; 371(s.n.): 75-84.

28. ROCCELLA EJ. Report of the National High Blood Pressure Education Program Working Group on High Blood Pressure in Pregnancy. American Journal of Obstetrics and Gynecology, 2000; 183(1): 1-22.

29. SOCIEDADE BRASILEIRA DE CARDIOLOGIA. Guidelines for pregnancy in the woman with heart disease. Arquivos brasileiros de cardiologia, 2009; 93(6) S1: e110-78.

30. THE PRISMA GROUP. Principais itens para relatar Revisões sistemáticas e Meta-análises: A recomendação PRISMA. Epidemiologia e Serviços de Saúde, 2015; 24(2): 335-342.

31. TOURINHO AB, REIS LBDSM. Birth Weight: A Nutricional Approach. Comunicação em Ciências da Saúde, 2013; 22(4): 19-30.

32. VAN OOSTWAARD MF, et al. Maternal and neonatal outcomes in women with severe early onset pre-eclampsia before 26 weeks of gestation, a case series. BJOG: An International Journal of Obstetrics and Gynaecology, 2017; 124(9): 1440-1447.

33. VATA PK, et al. Assessment of prevalence of preeclampsia from Dilla region of Ethiopia. BMC Research Notes, 2015; 8(1): 4-9.

34. VÁZQUEZ-RODRÍGUEZ JG, BARBOZA-ALATORRE DY. Maternal and perinatal outcomes of expectant treatment of severe preeclampsia. Revista medica del Instituto Mexicano del Seguro Social, 2018; 56(4): 379-386.

35. WAGNEW M, et al. Trends of preeclampsia/eclampsia and maternal and neonatal outcomes among women delivering in addis ababa selected government hospitals, Ethiopia: a retrospective cross-sectional study. The Pan African Medical Journal, 2016; 25(S2): 12.

36. WISNER K. Gestational Hypertension and Preeclampsia. MCN The American Journal of Maternal/Child Nursing, $2019 ; 44(3): 170$.

37. WORLD HEALTH ORGANIZATION (OMS). 2018. In: Care of the preterm and low-birth-weight newborn. Disponivel em: https://www.who.int/maternal_child_adolescent/newborns/prematurity/en/. Acesso em 05 de abril de 2019.

38. WORLD HEALTH ORGANIZATION (OMS). 2019. In: Maternal and perinatal health. Disponível em: https://www.who.int/maternal_child_adolescent/topics/maternal/maternal_perinatal/en/. Acesso em: 19 nov. 2019.

39. WORLD HEALTH ORGANIZATTION (OMS). Prevention and treatment of pre-eclampsia and eclampsia, 2011; [s.n.]. 